\title{
Multicode Chip-Interleaved DS-CDMA to Effect Synchronous Correlation of Spreading Codes in Quasi-Synchronous Transmission over Multipath Channels
}

\author{
Yu-Nan Lin and David W. Lin, Senior Member, IEEE
}

\begin{abstract}
The performance of conventional DS-CDMA systems is greatly affected by asynchronous or multipath propagation. We show that, with a certain way of multicode assignment, chip-interleaved DS-CDMA (CIDS-CDMA) can perform better in such a condition thanks to its ability to preserve the synchronous correlations among the spreading codes. Compared to the recently proposed chip-interleaved block-spread CDMA (CIBS-CDMA) that requires a single-user equalizer, the presented scheme can attain better or comparable performance in channel-coded transmission with a rake receiver that has lower complexity.
\end{abstract}

Index Terms-Chip interleaving, code division multiaccess, multipath channels.

\section{INTRODUCTION}

$\mathbf{T}$ HE capacity of a DS-CDMA system highly depends on the correlation properties of the spreading codes. Some well-known classes of spreading codes that have low inter-code correlations are the Gold, the Kasami, and the msequences. Their property of low inter-code correlations, however, may be lost in asynchronous transmission or multipath propagation, resulting in a performance close to that of random spreading [1].

By chip-interleaved block spreading (abbreviated CIBSCDMA) [2], [3], the orthogonality among spreading codes can be maintained in asynchronous or multipath propagation, and the multiaccess interference (MAI) is eliminated deterministically. Although intersymbol interference (ISI) will show up in multipath propagation, it can be dealt with using a single-user equalizer. However, an issue, heretofore unaddressed, arises when channel coding is employed. Briefly, if the residual ISI after equalization comes mainly from a small number of paths (in the equalized channel), then the performance of channel coding may suffer, because channel codes are normally designed for the white Gaussian noise condition whereas the residual ISI in this case is not white Gaussian. One remedy is to lengthen the equalizer for further ISI suppression. Another is to use turbo equalization that iterates

Manuscript received May 1, 2004; revised March 21, 2005; accepted April 14,2006 . The associate editor coordinating the review of this letter and approving it for publication was K. B. Lee. This work was supported by the National Science Council of the Republic of China under Grant NSC 92-2219-E-009-018.

Y.-N. Lin was with the Department of Electronics Engineering, National Chiao Tung University, Hsinchu, Taiwan, ROC. He is now with Realtek Semiconductor Corp., Hsinchu, Taiwan 300, ROC (e-mail: ynlin.ee87g@nctu.edu.tw).

D. W. Lin is with the Department of Electronics Engineering and Center for Telecommunications Research, National Chiao Tung University, Hsinchu, Taiwan 30010, ROC (e-mail: dwlin@mail.nctu.edu.tw).

Digital Object Identifier 10.1109/TWC.2006.04285 between equalization and channel decoding [4], [5]. Both entail additional complexity, with the turbo equalizer also increasing the decoding delay.

Herein we present a multicode chip-interleaved DS-CDMA (CIDS-CDMA) scheme, which can be viewed as a modification of CIBS-CDMA, that not only maintains the spreading codes' synchronous correlations (to be defined later) in quasi-synchronous multipath channels but also facilitates lowcomplexity rake receiving for channel-coded transmission. In what follows, we first describe the CIDS-CDMA signals in Section II. In Section III, we describe how the proposed scheme maintains the synchronous code correlation in quasisynchronous multipath propagation. We also give a system example that uses Gold sequences as the spreading codes. Section IV gives a brief comparison of the proposed scheme and CIBS-CDMA. Section V presents numerical results that illustrate the proposed scheme's performance. Finally, Section VI gives a conclusion.

\section{CIDS-CDMA SIGNALS}

\section{A. Transmitted Signal}

In CIDS-CDMA, the modulated signals are formed as as follows. First, data symbols are partioned into blocks. After spreading, the symbols in each block are transmitted with their chips interleaved. Whereas CIBS-CDMA employs one spreading code for each data block of a user, we allow use of multiple spreading codes. Its implications will become clear later.

Let each block of interleaved data contain $M_{d}$ symbols and, for the time being, assume that each symbol in the block is spread with a different code. Assume that $M_{0}$ zero chips are padded after each $M_{d}$ data chips as in [2], where $M_{0}$ may be null. Then the signal for block $g$ of user $k$ can be expressed as

$$
\underline{s}_{k}(g)=\sqrt{2 P} \mathbf{C}_{k} \underline{b}_{k}(g),
$$

where $\sqrt{2 P}$ is the normalized signal amplitude of each user, $\underline{b}_{k}(g)=\left[b_{k}\left(g M_{d}\right), \ldots, b_{k}\left((g+1) M_{d}-1\right)\right]^{T}$ denotes the $g$ th data block, and

$\mathbf{C}_{k}=\left[\operatorname{diag}\left(\underline{c}_{k}[0]\right), \mathbf{0}_{M_{d} \times M_{0}}, \ldots, \operatorname{diag}\left(\underline{c}_{k}[N-1]\right), \mathbf{0}_{M_{d} \times M_{0}}\right]^{T}$

is the "spreading matrix" with $N$ being the spreading factor, $\underline{c}_{k}[n](n=0, \ldots, N-1)$ the vector of the $n$th chips of the $M_{d}$ codes, and $\mathbf{0}_{M_{d} \times M_{0}}$ the all-zero matrix of size $M_{d} \times M_{0}$. The dimension of $\mathbf{C}_{k}$ is $N M \times M_{d}$ where $M=M_{d}+M_{0}$. The number of output chips for each block after spreading is thus equal to $N M$. 
Let $c_{k}^{(h)}[n](n=0, \ldots, N-1)$ denote the spreading code for the $h$ th data symbol of user $k$. Then, for block $g$,

$$
\underline{c}_{k}[n]=\left[c_{k}^{\left(g M_{d}\right)}[n], \ldots, c_{k}^{\left((g+1) M_{d}-1\right)}[n]\right]^{T},
$$

where the absence of index $g$ in the left-hand side reflects the fact that we use the same set of spreading codes for all blocks. (Change of spreading code assignment from block to block is not considered in this work. It may result in some performance gain whose amount depends on the operating condition, but the amount tends to be insignificant when users are many.) The synchronous correlation between the two codes for the $h^{\prime}$ th symbol of user $m$ and the $h$ th symbol of user $k$ is defined as

$$
\Lambda_{m, k}\left(h^{\prime}, h\right)=\sum_{n=0}^{N-1} c_{m}^{\left(h^{\prime}\right)}[n] c_{k}^{(h)}[n] .
$$

\section{B. Received Signal}

Let the maximum possible delay spread for any user be $L-1$ chips and the channel response for user $k$ be given by

$$
h_{k}[n]=\sum_{l=0}^{L-1} \alpha_{k}[l] \delta\left[n-\tau_{k}-l\right]
$$

where $\tau_{k}$ is the initial delay of user $k$ 's signal. The model accommodates both synchronous and quasi-synchronous transmission. For convenience, define $\alpha_{k}[l]=0$ for $l<0$ and $l \geq L$ and let $\tau_{\max }=\max _{k}\left\{\tau_{k}\right\}$. Let $M_{0} \geq \tau_{\max }+L-1$. After channel propagation, each block of user signal is spread over a time interval of no more than $N M+\tau_{\max }+L$ chips. In particular, the received signal in the interval $[g N M,(g+$ 1) $\left.N M+\tau_{\max }+L-2\right]$ (in unit of chips) due to user $k$ 's transmission can be expressed in vector form as

$$
\underline{x}_{k}(g)=\mathbf{H}_{k, 0} \underline{s}_{k}(g)+\mathbf{H}_{k,-1} \underline{s}_{k}(g-1)+\mathbf{H}_{k, 1} \underline{s}_{k}(g+1),
$$

where $\underline{x}_{k}(g)$ is an $\left(N M+\tau_{\max }+L-1\right)$-vector and $\mathbf{H}_{k, 0}$, $\mathbf{H}_{k,-1}$, and $\mathbf{H}_{k, 1}$ are $\left(N M+\tau_{\max }+L-1\right) \times N M$ Toeplitz matrices. Matrices $\mathbf{H}_{k, 0}$ and $\mathbf{H}_{k, 1}$ are lower triangular with their first columns given by $\left[\mathbf{0}_{1 \times \tau_{k}}, \alpha_{k}[0], \ldots, \alpha_{k}[L-1], 0, \ldots, 0\right]^{T}$ and $\left[0, \ldots, 0, \alpha_{k}[0], \ldots, \alpha_{k}\left[L+\tau_{\max }-\tau_{k}-2\right]\right]^{T}$, respectively, whereas $\mathbf{H}_{k,-1}$ is upper triangular with its first row given by $\left[0, \ldots, 0, \alpha_{k}[L-1], \ldots, \alpha_{k}[0], \mathbf{0}_{1 \times\left(\tau_{k}-1\right)}\right]$.

In (6), the second and the third right-hand-side terms are the interference from the previous and the subsequent data blocks and have been named interblock-interference (IBI). Thanks to zero padding, these IBI terms can be made of no influence to data detection, as already noted in [2]. Hence we may ignore them in subsequent derivation. Then the total received signal in the above time interval is given by

$$
\underline{x}(g)=\sum_{k=0}^{K-1} \underline{x}_{k}(g)+\underline{\xi}(g),
$$

where $\xi(g)=\left[\xi[g N M], \ldots, \xi\left[(g+1) N M+\tau_{\max }+L-2\right]\right]^{T}$ is a vector of additive noise samples, assumed white Gaussian.

\section{EfFecting Synchronous Code CorRelation After Multipath Propagation}

The rake receiver, which is of lower complexity than many other receiver structures, can be used to receive the above CIDS-CDMA signal. To fully collect the energy in the $l$ th path of user $k$ 's signal, the despreader input must contain the $\left(\tau_{k}+l\right)$ th to the $\left(N M-M_{0}-1+\tau_{k}+l\right)$ th elements of $\underline{x}(g)$. Denote by $\mathbf{H}_{k, 0}^{(l)}$ the matrix composed of the $l$ th to the $(N M+$ $l-1)$ th rows of $\mathbf{H}_{k, 0}$ and by $\xi^{(l)}(g)$ the vector consisting of the $l$ th to the $(N M+l-1)$ th elements of $\xi(g)$. Without loss of generality, consider detection of the 0 th user signal. Despreading of the received signal for the $l$ th path results in an $M_{d}$-vector given by

$$
\underline{y}_{0}^{(l)}(g)=\sqrt{2 P} \sum_{k=0}^{K-1} \mathbf{C}_{0}^{T} \mathbf{H}_{k, 0}^{\left(\tau_{0}+l\right)} \mathbf{C}_{k} \underline{b}_{k}(g)+\mathbf{C}_{0}^{T} \underline{\xi}^{\left(\tau_{0}+l\right)}(g) .
$$

Let $\mathbf{J}^{(h)}$ denote the $N M \times N M$ matrix whose elements are all zero except for the $h$ th diagonal where the elements are all ones, where $h=0$ refers to the main diagonal, $h>0$ a sub-diagonal, and $h<0$ a super-diagonal. $\left(\mathbf{J}^{(h)}\right.$ is an $h$-unit delay operator.) Then

$$
\begin{aligned}
\mathbf{C}_{0}^{T} \mathbf{H}_{k, 0}^{\left(\tau_{0}+l\right)} \mathbf{C}_{k} & =\mathbf{C}_{0}^{T} \cdot \sum_{d=0}^{L-1} \alpha_{k}[d] \mathbf{J}^{\left(\tau_{k}+d-\tau_{0}-l\right)} \mathbf{C}_{k} \\
& =\sum_{d=0}^{L-1} \alpha_{k}[d] \mathbf{C}_{0}^{T} \mathbf{J}^{\left(\tau_{k}+d-\tau_{0}-l\right)} \mathbf{C}_{k} .
\end{aligned}
$$

The matrix $\mathbf{C}_{0}^{T} \mathbf{J}^{\left(\tau_{k}+d-\tau_{0}-l\right)} \mathbf{C}_{k}$ is all zero except for the $\left(\tau_{k}+d-\tau_{0}-l\right)$ th diagonal. Let $(\mathbf{A})_{i, j}$ denote the $(i, j)$ th element of matrix $\mathbf{A}$. Then for $j=i-\left(\tau_{k}+d-\tau_{0}-l\right)$,

$$
\begin{aligned}
\left(\mathbf{C}_{0}^{T} \mathbf{J}^{\left(\tau_{k}+d-\tau_{0}-l\right)} \mathbf{C}_{k}\right)_{i, j} & =\sum_{n=0}^{N-1} c_{0}^{\left(g M_{d}+i\right)}[n] c_{k}^{\left(g M_{d}+j\right)}[n] \\
& =\Lambda_{0, k}\left(g M_{d}+i, g M_{d}+j\right) .
\end{aligned}
$$

Hence

$$
\mathbf{C}_{0}^{T} \mathbf{J}^{\left(\tau_{k}+d-\tau_{0}-l\right)} \mathbf{C}_{k}=\mathbf{J}^{\left(\tau_{k}+d-\tau_{0}-l\right)} \mathbf{D}^{\left(\tau_{k}+d-\tau_{0}-l\right)}
$$

where $\mathbf{D}^{(l)}$ is a diagonal matrix whose $i$ diagonal element is given by $\Lambda_{0, k}\left(g M_{d}+i, g M_{d}+i-l\right)$. In summary,

$$
\underline{y}_{0}^{(l)}(g)=\sqrt{2 P} \sum_{k=0}^{K-1} \mathbf{R}_{0, k}^{(l)} \underline{b}_{k}(g)+\underline{\xi}_{0}^{(l)}(g)
$$

where

$$
\begin{aligned}
\mathbf{R}_{0, k}^{(l)} & =\sum_{d=0}^{L-1} \alpha_{k}[d] \mathbf{J}^{\left(\tau_{k}+d-\tau_{0}-l\right)} \mathbf{D}^{\left(\tau_{k}+d-\tau_{0}-l\right)}, \\
\underline{\xi}_{0}^{(l)}(g) & =\mathbf{C}_{0} \underline{\xi}^{\left(\tau_{0}+l\right)}(g) .
\end{aligned}
$$

From the above, it is clear that the despreading result is entirely determined by the synchronous correlation of the spreading codes although the transmission is over a multipath channel. Thus, unlike conventional DS-CDMA whose performance depends on both the even and the odd code correlations [6], the multicode CIDS-CDMA has a more controllable performance. 


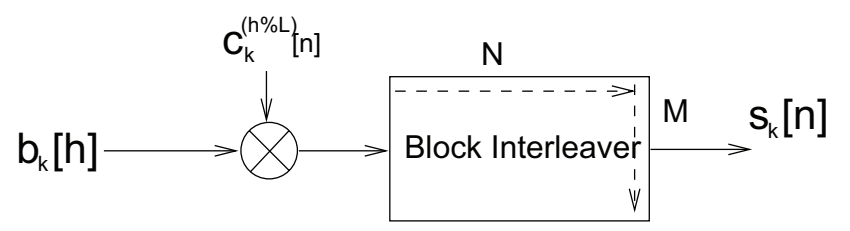

Row Input, Column Output

Fig. 1. A CIDS-CDMA transmitter that employs $L$ spreading codes periodically.

The rake combiner output for $\underline{b}_{0}(g)$ is given by

$$
\underline{\hat{b}}_{0}(g)=\sum_{l=0}^{L-1} \alpha_{0}^{*}[l] \underline{y}_{0}^{(l)}(g),
$$

which contains ISI, MAI, and noise, in addition to the desired signal $\underline{b}_{0}(g)$.

Since the number of spreading codes having low synchronous correlation is limited, we should conserve code usage. From the above discussion, we can see that it suffices to have $L$ spreading codes for each user, to be used periodically, when the multipath delay spread is not greater than $L-1$ chips. This is illustrated in Fig. 1.

\section{A. An Example Using Gold Sequences}

Any set of sequences with low (including zero) synchronous correlation can be used in the proposed system. However, all types of sequences are not equal considering system capacity. For example, only $N / L$ users can be accommodated if WalshHadamard sequences are used, but other sequences may permit more. A recent paper presents optimal binary sequence design for many combinations of processing gain and number of used spreading sequences [7]. However, to change the number of used sequences may require a redesign of all used sequences. In addition, Gold sequences are shown to be optimal in various conditions [7]. Hence we consider use of Gold sequences for reasons of flexibility and performance.

A set of Gold sequences of length $N$ is constructed from a preferred pair of m-sequences of length $N$. The set contains $N+2$ sequences, including the preferred pair. Dropping one of the two $\mathrm{m}$-sequences from the set results in a set where the correlation value between any two sequences is -1 . This property is used in our code assignment strategy.

Unlike conventional spreading code assignment, we assign the spreading codes gluttonously as follows. We assign $L$ of the $N+1$ Gold sequences to the first user, then another $L$ to the second user, and so on, until we exhaust the $N+1$ sequences. Then we assign the $N+1$ sequences which are one cyclic shift of the original Gold sequences, and then those which are two cyclic shifts, and so on. This way, we can assure that in the despreading at each rake finger, there are always $N$ among the $K L-1$ interfering signals that have a code correlation equal to -1 . Numerical evaluation shows that the mean-square synchronous correlation of cyclically shifted Gold sequences is close to $N$, similar to random codes. Hence, by Gaussian approximation, the resultant bit error rate (BER) in random static channels when the multipaths are all of equal strength is given by

$$
P_{\mathrm{CI}}= \begin{cases}Q\left(\sqrt{\left.\frac{1}{\frac{1}{S N R}+\frac{N}{2 N^{2} L}+\frac{K L-N-1}{2 N L}}\right),}, \quad K L>N+1,\right. \\ Q\left(\sqrt{\left.\frac{1}{\frac{1}{S N R}+\frac{K L-1}{2 N^{2} L}}\right),},\right. & K L \leq N+1 .\end{cases}
$$

In comparison, the performance of conventional DS-CDMA under random-code spreading is given by

$$
P_{\text {conv }}=Q\left(\sqrt{\frac{1}{\frac{1}{S N R}+\frac{K L-1}{2 N L}}}\right) .
$$

Comparing (15) with (16), it is clear that the performance of the proposed system is always better than the conventional one, especially when $K L$ is not much larger than $N+1$.

\section{COMPARISON WITH CIBS-CDMA}

As discussed previously, CIBS-CDMA assigns one spreading code to each user, where the spreading codes are orthogonal. This deterministically eliminates MAI in multipath propagation, but results in ISI which can be dealt with by singleuser equalization. In comparison, the proposed scheme may employ orthogonal or nonorthogonal codes and it may assign one or more codes to a user, depending on the desired capacity and the channel condition. The proposed code assignment has several advantages.

First, for CIBS-CDMA, the residual ISI after single-user equalization may be far from being Gaussian when the equalized channel consists of only a small number of taps. Such ISI may highly degrade the performance of channel coding as can be seen in the numerical results of various studies [4], [5], [8], [9]. With multicode CIDS-CDMA, the total interference approaches Gaussian more closely due to the presence of a larger number of interferers (consisting of the different user signals, spread with multiple spreading codes and propagated over multipath channels). This is beneficial to the error correction capability of channel coding.

Second, the proposed multicode CIDS-CDMA provides a soft capacity as conventional DS-CDMA does, in the sense that the performance degrades gracefully as more users are accommodated. In contrast, the capacity of CIBS-CDMA is limited by the number of orthogonal sequences. (One could consider overloading the CIBS-CDMA system with additional users whose signals are spread with nonorthogonal spreading sequences. But then the added users may experience performance similar to that of conventional DS-CDMA due to MAI.)

Third, the proposed scheme offers added flexibility in leveraging between receiver complexity and transmission performance. For low complexity, a rake receiver may be employed. Table I shows the relative complexity of some core functions in the rake receiver and the CIBS-CDMA equalizer in detecting one user symbol. In multicode CIDS-CDMA, assume that a user is assigned $L$ spreading codes and the rake receiver has (at most) $L$ fingers. The rake receiver needs to despread up to $L$ codes per user symbol, but the CIBS-CDMA equalizer only one. However, despreading is usually of lower complexity compared to the other functions in Table I. For CIBS-CDMA, the equalizer complexity depends on its type. Table I lists the complexity involved with serial and block linear MMSE 
TABLE I

RElative COMPLEXITy OF RAKE RECEIVER FOR MUlTicode CIDS-CDMA AND EQUALIZER FOR CIBS-CDMA

\begin{tabular}{|l|c|c|c|}
\hline & Despreading & Filtering & Filter Computation* \\
\hline CIDS-CDMA & $L$ & $L$ & 0 \\
\hline CIBS-CDMA (serial) & 1 & $L_{g}$ & $\left(L+L_{g}+1\right)^{2} / M_{d}$ \\
\hline CIBS-CDMA (block) & 1 & $M_{d}+M_{0}$ & $\left(M_{d}+M_{0}\right)^{2} / M_{d}$ \\
\hline
\end{tabular}

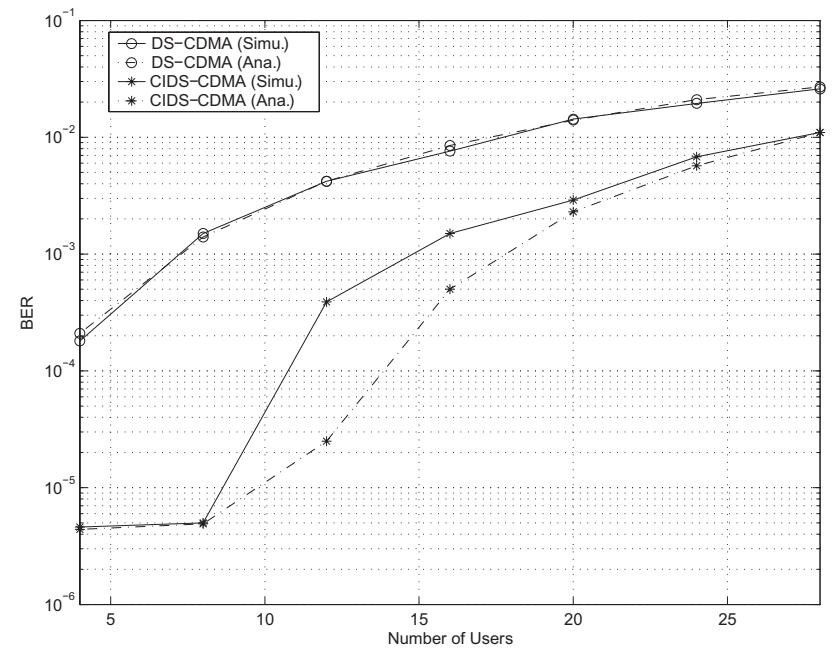

Fig. 2. BER performance of conventional DS-CDMA and CIDS-CDMA under Gold-sequence spreading, for different user numbers at $L=6, N=$ 63 , and $\mathrm{SNR}=13 \mathrm{~dB}$.

equalization employing the low-complexity method to compute the equalizer coefficients [2], where $L_{g}$ is the number of taps for serial equalizer. To have better transmission efficiency $\left(=M_{d} /\left(M_{d}+M_{0}\right)\right), M_{d}$ should be much larger than $M_{0}$. Hence, block equalization is not suitable in such a system due to the huge demand of equalizer taps. For serial equalization, experience shows that we usually need $L_{g}>L$ to have good results. Hence the proposed scheme will normally have a lower receiver complexity. Now Table I does not count in the computations needed of the signal correlations for determining the rake finger weights or that for setting up the equation for calculating the CIBS-CDMA equalizer coefficients. However, their relative complexity should be on the order of $L$ versus $L_{g}$. On the other hand, if better performance than what rake can provide is desired in a multicode CIDS-CDMA system, an equalizer can also be employed. This is especially the case when the number of spreading codes assigned to the concerned user is smaller than $L$. This equalizer can have a lower complexity than that in CIBS-CDMA [10]. The details are omitted here.

\section{Simulation Results}

\section{A. Comparison With Conventional DS-CDMA}

Firstly, we present results for random static channels. We let each initial delay $\tau_{k}$ be uniformly distributed in $\{0,1, \ldots, N-1\}$. Limiting $\tau_{\max }$ to $N-1$ is for easier comparison between conventional DS-CDMA and multicode CIDS-CDMA; it is not a fundamental limit to the proposed scheme. The multipaths have the same amplitude, with their

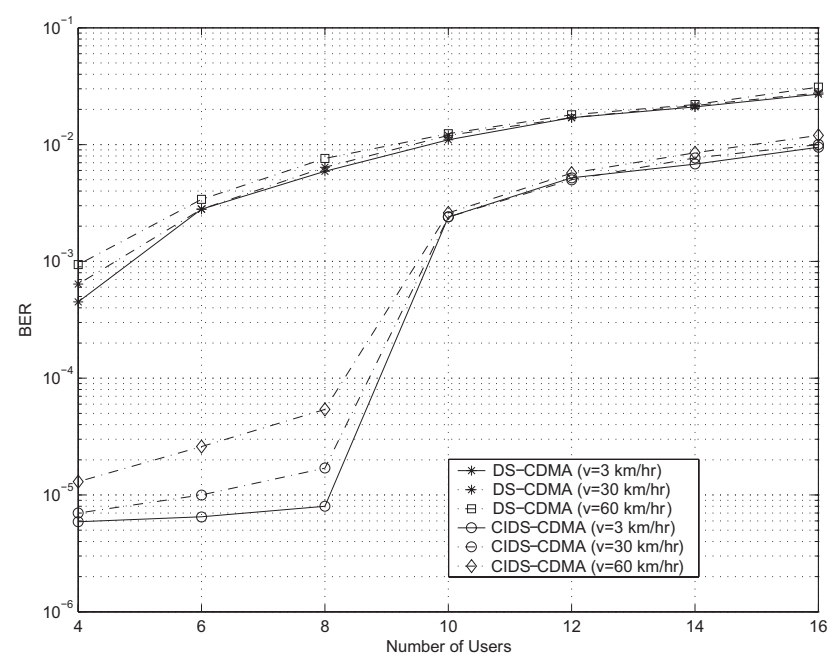

Fig. 3. BER performance of conventional DS-CDMA and CIDS-CDMA under Gold-sequence spreading, for different user numbers at $L=4, N=$ 31 , and $\mathrm{SNR}=13 \mathrm{~dB}$ under fading channels.

phases uniformly distributed in $[0,2 \pi)$. Gold sequences are used in both systems. Fig. 2 shows the results for $N=63$ and $L=6$ at $\mathrm{SNR}=13 \mathrm{~dB}$. As pointed out in [1], the results for conventional DS-CDMA under asynchronous transmission match closely the theoretical result for random-code spreading (given in (16)). With the proposed code assignment, CIDSCDMA clearly outperforms conventional DS-CDMA especially when the user number is small. The larger discrepancy between the simulation results and the theory at when $K$ is about $(N+1) / L$ can be explained by noting that, because the number of spreading codes with different cyclic shifts is relatively small in this situation, the MAI has a greater variance about its mean than when $K$ is larger.

The foregoing analysis and simulation have assumed that the channel responses remain unchanged during the whole chip-interleaving block so as to effect perfect synchronous correlation among the spreading codes. Therefore, question arises as to how multicode CIDS-CDMA performs in fading channels. Hence we consider transmission over multipath Rayleigh fading channels next. Let $N=31, M_{d}=152$, $M_{0}=12$, carrier frequency $=2 \mathrm{GHz}$, and chip rate $=3.84$ Mcps (as in 3GPP [11]). Assume that $\tau_{\max }=8$ chips, which corresponds to a maximum $625 \mathrm{~m}$ difference in initial path lengths, and asssume that $L=4$. For simplicity, let the total path energy be normalized per chip-block length, which is $31 \times 164=5084$ chips and about two slots in 3GPP. The results for different moving speeds are shown in Fig. 3. As can be expected, channel fading rate has minor impact on DSCDMA since we have assumed quite accurate power control. Comparatively, faster fading does more harm to CIDS-CDMA because channel fading alters the correlation among users. Nevertheless, under the simulated parameters, CIDS-CDMA still outperforms DS-CDMA with slight capacity loss.

\section{B. Comparison With CIBS-CDMA}

Now we simulate channel-coded transmission where the channel code is the rate-1/2 binary convolutional code of constraint length 7 with generators $g_{1}=133_{8}$ and $g_{2}=171_{8}$. 


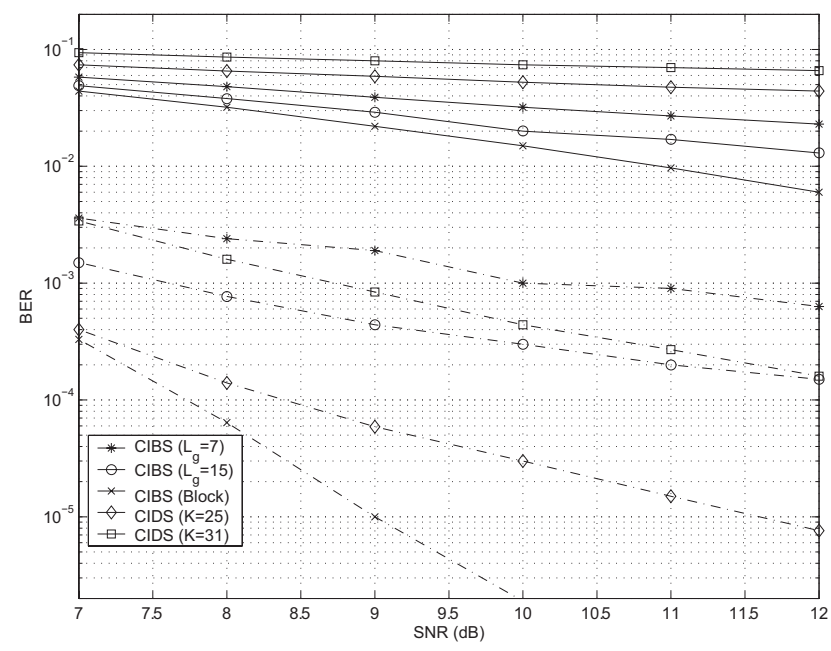

Fig. 4. BER performance of convolutionally coded CIBS-CDMA and CIDSCDMA as a function of SNR with $N=32$ and 31, respectively. Solid lines: before channel decoding; dashed lines: after channel decoding.

And we employ soft-decision Viterbi decoding with traceback length $=5 \times 7=35$. For spreading, we use Gold sequences of length 31 in multicode CIDS-CDMA and Walsh-Hadamard sequences of length 32 in CIBS-CDMA. In both systems, $M_{d}=144$ and $M_{0}=4$. A $24 \times 24$ block bit-interleaver is inserted between the channel coder and the spectrum spreader to disperse the sometimes bursty errors for better decoding performance. We simulate synchronous transmission over 4path channels with average path energies $0,-1,-2$, and $-3 \mathrm{~dB}$. The path coefficients are Rayleigh distributed. Each simulation run involves one randomly generated channel. In each run, we simulate perfect power control by normalizing the total path energy. Fig. 4 shows the average results over 1,000 simulation runs. For CIBS-CDMA, the serial equalization delay is set to $\left\lceil L_{g} / 2\right\rceil$. Observe that although CIBSCDMA with serial equalization has better performance before channel decoding, its performance after decoding improves less dramatically than multicode CIDS-CDMA due to nonGaussian distribution of the residual ISI. Even under the very heavy system loads simulated, the latter can still outperform the former at a lower receiver complexity. CIBS-CDMA with block equalizer is also shown there and demonstrates a superior performance especially in high SNR. However, the required taps for equalization is 148 , which is too complex to be implemented. The performance of CIDS-CDMA can be further improved by applying some advanced interference cancellation techniques. However, it is beyond of the scope of this letter.

\section{CONCLUSION}

We proposed a multicode chip-interleaved DS-CDMA scheme for transmission over slow-fading multipath channels. Through analysis and simulation, we showed that the proposed scheme had superior performance to conventional DS-CDMA in this condition, thanks to its ability to preserve the synchronous correlation among the spreading codes. In addition, the scheme was able to attain comparable or better performance than CIBS-CDMA at a lower receiver complexity, even under a heavy system load.

\section{REFERENCES}

[1] K. H. A. Karkkainen and P. A. Leppanen, "Comparison of the performance of some linear spreading code families for asynchronous DS/SSMA systems," in Proc. IEEE Military Commun. Conf., Nov. 1991, pp. 784-790.

[2] S. Zhou, G. B. Giannakis, and C. Le Martret, "Chip-interleaved blockspread code division multiple access," IEEE Trans. Commun., vol. 50, no. 2, pp. 235-248, Feb. 2002.

[3] S. Zhou, P. Xia, G. Leus, and G. B. Giannakis, "Chip-interleaved blockspread CDMA versus DS-DMA for cellular downlink: A comparative study," IEEE Trans. Wireless Commun., vol. 3, no. 1, pp. 176-190, Jan. 2004.

[4] A. Picart, P. Didier, and A. Glavieux, "Turbo-detection: A new approach to combat channel frequency selectivity," in Proc. IEEE Intl. Conf. Commun., June 1997, pp. 1498-1502.

[5] C. Laot, A. Glavieux, and J. Labat, "Turbo equalization: Adaptive equalization and channel decoding optimized," IEEE J. Select. Areas Commun., vol. 19, no. 9, pp. 1744-1752, Sep. 2001.

[6] M. B. Pursley, "Performance evaluation for phase-coded spreadspectrum multiple-access communication-Part I: System analysis," IEEE Trans. Commun., vol. 25, no. 8, pp. 795-799, Aug. 1977.

[7] G. N. Karystinos and D. A. Pados, "New bounds on the total squared correlation and optimum design of DS-CDMA binary signature sets," IEEE Trans. Commun., vol. 50, no. 1, pp. 48-51, Jan. 2003.

[8] M. V. Eyuboğlu and S. U. H. Qureshi, "Reduced-state sequence estimation for coded modulation on intersymbol interference channels," IEEE J. Select. Areas Commun., vol. 7, no. 6, pp. 989-995, Aug. 1989.

[9] F. Xiong, "Sequential decoding of convolutional codes in channels with intersymbol interference," IEEE Trans. Commun., vol. 43, no. 2/3/4, pp. 828-836, Feb./Mar./Apr. 1995.

[10] Y.-N. Lin and D. W. Lin, "A chip-interleaved synchronous DS-CDMA technique enabling MAI-free and reduced-ISI transmission with low complexity receiving," in Proc. IEEE Workshop Signal Processing Advances Wireless Commun., July 2004, pp. 487-491.

[11] 3GPP, "Technical specification group radio access network; Spreading and modulation (FDD),” Doc. 3G TS 25.213 ver. 4.1.0, June 2001. 